OPEN ACCESS

Edited by:

Danièle Werck,

UPR2357 Institut de biologie moléculaire des plantes (IBMP), CNRS, France

Reviewed by: Koichi Kobayashi, Osaka Prefecture University, Japan

Thomas J. Bach,

Université de Strasbourg, France

*Correspondence:

Venkatasalam Shanmugabalaji shanmugabalaji.venkatasalam@unine.ch

Specialty section: This article was submitted to Plant Metabolism and Chemodiversity, a section of the journal

Frontiers in Plant Science

Received: 01 November 2019 Accepted: 01 January 2020 Published: 31 January 2020

Citation:

Shanmugabalaji V, Grimm B and Kessler $F$ (2020) Characterization of a

Plastoglobule-Localized

SOUL4 Heme-Binding Protein in Arabidopsis thaliana.

Front. Plant Sci. 11:2.

doi: $10.3389 /$ fpls.2020.00002

\section{Characterization of a Plastoglobule- Localized SOUL4 Heme-Binding Protein in Arabidopsis thaliana}

\author{
Venkatasalam Shanmugabalaji ${ }^{1 *}$, Bernhard Grimm ${ }^{2}$ and Felix Kessler ${ }^{1}$ \\ ${ }^{1}$ Laboratory of Plant Physiology, Institute of Biology, University of Neuchâtel, Neuchâtel, Switzerland, ${ }^{2}$ Institute of Biology/ \\ Plant Physiology, Humboldt University Berlin, Berlin, Germany
}

Heme plays an active role in primary plant metabolic pathways as well as in stress signaling. In this study, we characterized the predicted heme-binding protein SOUL4. Proteomics evidence suggests that SOUL4 is a component of Arabidopsis plastoglobules (PGs, chloroplast lipid droplets). SOUL4 contains heme-binding motifs and the recombinant protein is shown here to bind heme in vitro. Fluorescence-tagged SOUL4 colocalized with the specific PG marker Fibrillin1A (FBN1A) in transiently transformed Nicotiana benthamiana leaves. In addition, SOUL4 cofractionated with another PG marker Fibrillin2 (FBN2) in sucrose gradient ultracentrifugation experiments. In vitro kinase experiments revealed that SOUL4 is phosphorylated by a yet unknown chloroplast protein kinase. Our data demonstrate that SOUL4 is a bona fide PG protein and may function in heme-buffering in the chloroplast.

Keywords: plastoglobule, SOUL4, heme-binding protein, chloroplast, kinase

\section{INTRODUCTION}

Tetrapyrroles play key roles in photosynthesis, respiration, and in various other biological processes. In plants, chlorophyll, heme, and phytochromobilin ( $\mathrm{P} \Phi \mathrm{B})$ are the main tetrapyrroles derived from a common biosynthetic pathway in chloroplasts (Mochizuki et al., 2010). Due to their photochemical properties, misregulation of tetrapyrrole metabolism may lead to severe photooxidative damage and cell death (Busch and Montgomery, 2015). Thus, tetrapyrrole transport and metabolism need to be tightly controlled (Brzezowski et al., 2015; Kobayashi and Masuda, 2016). Protoporphyrin IX serves as a common intermediate in both the chlorophyll- and heme-synthesized branches of tetrapyrrole metabolism. In the heme branch, ferrochelatase inserts $\mathrm{Fe} 2+$ into protoporphyrin IX to form heme. Heme is further degraded biliverdin IX $\alpha$ by heme oxygenase. In plants, biliverdin IX $\alpha$ can be reduced by phytochromobilin synthase to phytochromobilin, which is the chromophore of the phytochrome photoreceptor family (Mochizuki et al., 2010). The intracellular trafficking of heme from the chloroplasts to the cytosol, mitochondria, as well as other organelles remains to be elucidated. Oxidative stress and ABA perception lead to increased heme content in the cell (Vanhee et al., 2011). Accumulating heme molecules are capable of reacting with oxygen to generate cytotoxic ROS (Busch and Montgomery, 2015). To prevent this, a number of heme-binding proteins exist in various cellular compartments. It is proposed that these proteins protect the cell from the cytotoxic effects and bind to heme covalently and/or noncovalently (Balestrasse et al., 2005; Espinas et al., 2012). 
Heme-binding proteins may act as carriers, transporters, or regulators of the remaining free heme pool. In plants, tryptophan-rich sensory protein (TSPO) is a well-known heme-binding protein present in most subcellular compartments (Veenman et al., 2016). Under oxidative stress, excess heme binds to TSPO and interacts with autophagy-related protein 8 (ATG8) for further degradation through the autophagy pathway (Vanhee et al., 2011). Furthermore, Arabidopsis SOUL heme-binding protein 5 (SOUL5) has been identified as a hemebinding protein that interacts with heme oxygenase in the chloroplast (Lee et al., 2012).

A first SOUL protein was reported in the chicken retina and pineal gland, specifically expressed in response to light signaling (Zylka and Reppert, 1999). The SOUL protein uses a His-residue as an axial ligand to coordinate the $\mathrm{Fe}(\mathrm{II})$-containing heme. Heme-binding triggers the formation of hexameric SOUL complexes (Sato et al., 2004; Dias et al., 2006). Five SOUL isoforms have been annotated in Chlamydomonas reinhardtii (Merchant et al., 2007). The Chlamydomonas SOUL3 homolog has been implicated in the organization and positioning of the eyespot within the cell and may play a role in algal light perception (Schulze et al., 2013). In Arabidopsis, there are five proteins with a SOUL motif. In vitro studies demonstrated that SOUL1 (At1g17100) and SOUL2 (At2g37970) are cytosolic and bind to heme as well as other porphyrins (Takahashi et al., 2008). The proteome of the Arabidopsis chloroplasts revealed the presence of SOUL5 at the thylakoid membrane (Peltier et al., 2006) which was further confirmed in a localization study (Lee et al., 2012). The SOUL4 protein (At3g10130) was identified in the proteome of plastoglobules (PGs), another chloroplast subcompartment (Vidi et al., 2006; Ytterberg et al., 2006; Lundquist et al., 2012).

PGs are lipoprotein particles within the chloroplast. PGs are bounded by a membrane lipid monolayer that is contiguous with the outer leaflet of the thylakoid membrane. The interior space of PG is filled with hydrophobic neutral lipids such as plastoquinone, phylloquinone, $\alpha$-tocopherol, fatty acid phytyl esters, triacylglycerol, and carotenoids (Kessler et al., 1999; Austin et al., 2006). The first PG-associated protein was discovered in red pepper chromoplasts that contain carotenoid-rich fibrils which are structurally related to PG (Deruère et al., 1994). Hence the members of the newly discovered family of proteins were named fibrillins (FBNs). PG-associated FBNs were identified in many species of plants, algae, and cyanobacteria (Deruère et al., 1994; Pozueta-Romero et al., 1997; Kessler et al., 1999). PGs have functional roles in stress responses, thylakoid breakdown, and chloroplast development (Van Wijk and Kessler, 2017). The proteome of PGs has around 30 proteins, which fall into three categories: FBNs, plastid metabolic proteins [such as tocopherol cyclase (VTE1)], and unknown proteins (Lundquist et al., 2012). Their protein composition suggests that chloroplast PG are heavily involved in prenyllipid and anti-oxidant metabolism protecting thylakoid membranes from ROS-induced damage. In senescing and stressed chloroplasts, the thylakoid membrane is dismantled and chlorophyll is broken down. In these processes, fatty acids (from galactolipids) and phytol (from chlorophyll) are liberated. Membrane-perturbing phytol is esterified with a free fatty acid by PG-localized phytyl ester synthases (Lippold et al., 2012) resulting in non-toxic phytol esters that are deposited inside PG.

In this study, we characterized SOUL4 using a combined biochemical and reverse genetic approach. The data show that SOUL4 is a heme-binding protein. We provide new evidence that SOUL4 is located in chloroplast PG and that it is phosphorylated by an unknown chloroplast kinase.

\section{RESULTS}

\section{SOUL4 Is Imported Into Chloroplast and Localized at PGs}

Three independent proteomics studies indicate that SOUL4 is mainly present in PGs and may constitute up to $1.8 \%$ of the total PG proteome mass (Lundquist et al., 2012). The open reading frame of the SOUL4 cDNA predicts a protein of 309 amino acids including a predicted chloroplast transit peptide of 72 amino acids at the N-terminus as well as the SOUL heme-binding motif. We further analyzed whether the SOUL4 protein was imported into the chloroplast, whether its predicted transit peptide was processed, and whether SOUL4 is targeted to PGs. The SOUL4 preprotein was synthesized in a coupled transcription/translation system in the presence of $\left[{ }^{35} \mathrm{~S}\right]$ methionine and incubated with isolated Arabidopsis chloroplasts in vitro. Two major radioactively labeled proteins were detected: one with an apparent molecular mass (MM) of around $35 \mathrm{kDa}$ corresponding to the expected $\mathrm{MM}$ of the in vitro synthesized SOUL4 preprotein and the second band with an apparent $\mathrm{MM}$ of around $30 \mathrm{kDa}$ corresponding to the mature form of SOUL4 (Figure 1A) (Venkatasalam, 2012). After reisolation and treatment of the chloroplasts with thermolysin, most of the $35 \mathrm{kDa}$ polypeptide was digested, whereas the $30 \mathrm{kDa}$ protein was largely protected against proteolytic attack. This shows that the SOUL4 preprotein was imported into the chloroplast and processed to its mature form. To address targeting of SOUL4 to PG, we engineered a construct encoding a SOUL4-CFP fusion under the control of the cauliflower mosaic virus (CaMV) 35S promoter. The SOUL4-CFP construct was transformed into Nicotiana benthamiana leaves by agroinfiltration and analyzed by confocal laser scanning microscopy. The SOUL4-CFP fluorescence was present in punctate structures reminiscent of PG (Figure 1B), but was detected neither in the thylakoid nor the envelope membranes (Venkatasalam, 2012). To determine whether SOUL4 colocalized with the PG marker FBN1A, the two proteins were co-expressed as CFP and YFP fusion proteins in N. benthamiana leaves by agroinfiltration. In this experiment, SOUL4-CFP as well as FBN1A-YFP fluorescence was present in the punctate structures inside chloroplasts. Both the merge of the independent fluorescence images and the pixel intensity analysis showed a signal overlap, indicating colocalization of SOUL4 and FBN1A in PGs (Figure 1C) (Venkatasalam, 2012). In addition, overexpression of SOUL4-YFP in stable transgenic 
A

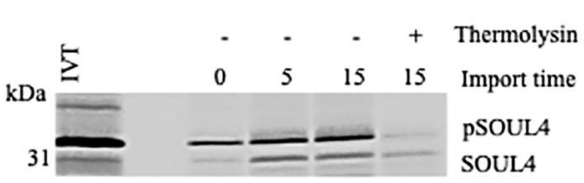

B
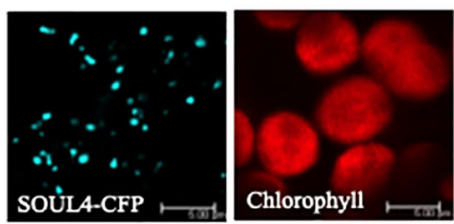

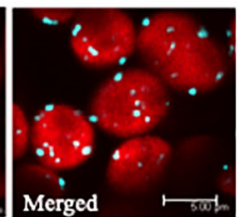

C
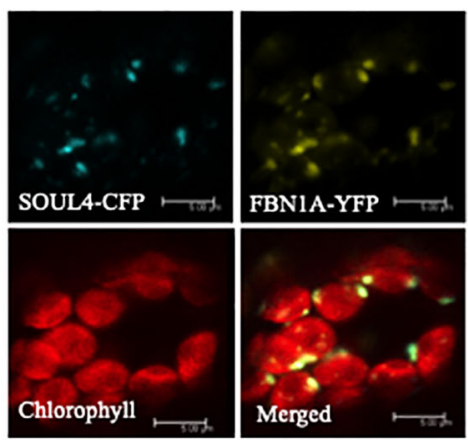

D

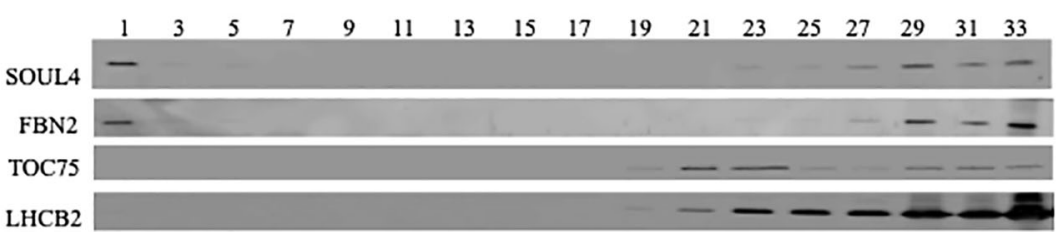

FIGURE 1 | Localization of the SOUL4 heme-binding protein in chloroplast plastoglobules. (A) Import of AtSOUL4 preprotein into isolated chloroplasts. In vitro synthesized [35S] SOUL4 preprotein (pSOUL4) was incubated with isolated Arabidopsis chloroplasts in a time course experiment. After reisolation and thermolysin treatment, the samples were analyzed by SDS-PAGE followed by autoradiography. pSOUL4 (35 kDa) before and mature protein SOUL4 (30 kDa) after thermolysin treatment are indicated. (B) Transient expression of SOUL4-CFP in N. benthamiana leaves. Fluorescent proteins were visualized by confocal laser scanning microscopy $48 \mathrm{~h}$ after agroinfiltration. Chlorophyll, CFP, and merge indicate chlorophyll autofluorescence, CFP fluorescence, and the superposition of both fluorescent signals, respectively. Bar length: $5 \mu \mathrm{m}$. (C) Colocalization of SOUL4-CFP and FBN1A-YFP. The SOUL4-CFP and FBN1A-YFP were co-expressed transiently in leaves of $N$. benthamiana, and localization was examined by fluorescence microscopy. All images were taken at the same magnification. Chlorophyll: chlorophyll autofluorescence, merge: superposition of YFP and CFP signals (yellow green color). The scale bar represents $5 \mu$ m. (D) Western blot detection of SOUL4 in chloroplast membrane fractions. After sucrose gradient floatation of total chloroplast membranes, fractions were collected starting from the top of the gradient. Equal volumes of fractions 1-33 were separated by SDS-PAGE and used for immunoblotting with antibodies against SOUL4, FBN2, TOC75 and LHCB2.

plants gave punctate fluorescence pattern inside chloroplasts (see Supplementary Figure 1). To obtain physical evidence of PG association, total membranes of isolated chloroplasts were fractionated on a sucrose gradient. This resulted in the separation of membrane fractions corresponding to broad and partially overlapping peaks of marker proteins by immunoblotting analysis. Antibodies against LHCB2 gave strong signals in fractions 23-33 (Figure 1D), indicating the presence of thylakoid membranes in the high density sucrose fractions. TOC75, a protein import channel of the outer envelope membrane, was mainly detected in fractions 21-23 (Figure 1D). SOUL4 co-distributed with FBN2 in the lowest density fraction (Figure 1D) (Venkatasalam, 2012). Presence of SOUL4 and FBN2 in the higher density fractions is in part due to incomplete separation of PG from thylakoid membranes and in part to bona fide partitioning between PG and thylakoid membranes (Lundquist et al., 2012).

\section{The SOUL4 Protein Binds Heme In Vitro}

Structural studies of SOUL protein revealed a novel hydrophobic cleft flanked by an $\alpha$-helix and the $\beta 8-\beta 9$ loop that was essential for heme-binding (Dias et al., 2006). All five Arabidopsis thaliana homologs have this novel hydrophobic cleft for heme-binding as it has been already experimentally demonstrated for AtSOUL1 and AtSOUL2 (Takahashi et al., 2008). Mature SOUL4 was expressed in Escherichia coli, purified as a soluble protein, and confirmed by immunoblotting. To investigate heme-binding properties, the purified recombinant SOUL4 was incubated with hemin-agarose and agarose alone as a negative control. Input, flow-through, last wash, and eluate fractions were analyzed by immunoblotting. SOUL4 bound to hemin-agarose and was detected in the eluate fraction (Figure 2A, left panel, lane $\mathrm{E}$ ), while it was not found in eluate fraction of the agarose alone (Figure 2A, right panel, lane E) (Venkatasalam, 2012). To exclude non-specific binding to hemin-agarose, a heme competition assay was carried out. SOUL4 (100 $\mu \mathrm{M})$ was incubated with $10 \mathrm{mM}$ hemin. After the incubation, the reaction mixture was applied to the hemin-agarose and subsequently washed and eluted. The load, flow-through, and wash fractions as well as the eluate were separated by SDS-PAGE followed by immunoblot analysis. The first wash removed most of the non-bound SOUL4, and the eluate contained very little SOUL4, demonstrating that SOUL4 binding to hemin-agarose was strongly impaired after incubation with $10 \mathrm{mM}$ hemin (Figure 2B) (Venkatasalam, 2012). Together, the data indicate that SOUL4 specifically binds heme in vitro. 
$\mathbf{A}$

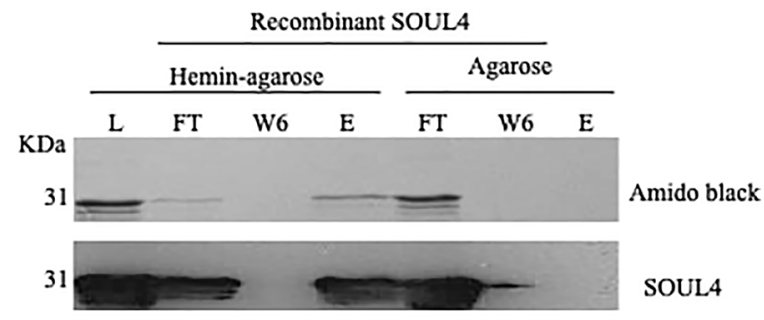

B

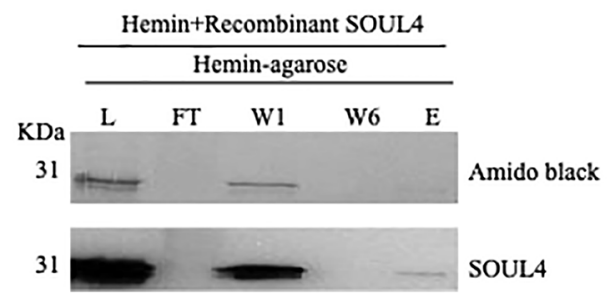

FIGURE 2 | In vitro heme-binding by mature SOUL4. (A) Binding of recombinant mature SOUL4 to hemin-agarose or agarose alone, as a negative control. The load (L), flow-through (FT), wash 6 (W6), and eluted (E) fractions were analyzed by SDS-PAGE, transferred to a nitrocellulose membrane, and stained with Amido black (upper panel). Antibodies against SOUL4 were used for immunoblotting, as indicated (lower panels). (B) SOUL4 binding to hemin-agarose in the presence of $10 \mathrm{mM}$ added soluble hemin. The load (L), wash 1, wash 6 (W1, W6), and eluted (E) fractions were analyzed by SDS-PAGE, transferred to a nitrocellulose membrane, and stained with Amido black (upper panel). Antibodies against AtSOUL4 were used for immunoblotting as indicated (lower panels).

\section{Characterization of T-DNA Insertion Mutant of SOUL4}

We isolated Arabidopsis SOUL4 T-DNA insertion lines. The soul4-1 insertion line contained T-DNA insertions in intron 2 and soul4-2 insertion line contained T-DNA insertions in $\mathbf{5}^{\prime}$ untranslated region (5' UTRs) (Figure 3A) (Venkatasalam, 2012). Homozygous lines were obtained by selfing and verified by segregation analysis and genotyping. The SOUL4 protein was not immunologically detected in total leaf extracts of soul4-1 confirming a knockout genotype of nature of the T-DNA insertion (Figure 3C) (Venkatasalam, 2012). Under standard growth conditions on soil, homozygous soul4-1 and soul4-2 grew normally and exhibited a wild-type phenotype (Figure 3B) (Venkatasalam, 2012). Earlier studies had shown that a number of PG proteins [e.g. VTE1 and $\mathrm{NAD}(\mathrm{P}) \mathrm{H}$ dehydrogenase $\mathrm{C} 1$ (NDC1)] are involved in prenylquinone metabolism (Vidi et al., 2006; Eugeni Piller et al., 2011). To determine whether SOUL4 plays a role in prenylquinone metabolism, untargeted analyses as well as targeted prenylquinone profiling were carried out. The clustering of the soul4-1 and WT-derived metabolomes revealed no differences by principal component analysis of either untargeted lipidomics or targeted prenylquinone profiling data (data not shown) indicating that the two genotypes have largely identical metabolomes under standard growth conditions. Phylogenetic analysis of Arabidopsis SOUL proteins revealed that SOUL5 was a close homolog of SOUL4 (see Supplementary Figure 2) and moreover, SOUL5 is also localized in the chloroplast. To investigate the role of two chloroplast SOUL proteins in tetrapyrrole accumulation, we also isolated the soul5 and soul41/5 mutants (see Supplementary Figure 3). In higher plants, the chlorophyll- and heme-synthesizing pathways play essential roles in cellular and organellar function. We measured the content of chlorophyll $a$ and $b, \mathrm{Mg}$-protoporphyrin IX, and heme from wild type, soul4-1, soul5, soul4-1/5, and SOUL4-YFP overexpressing lines (SOUL4-YFP1 and SOUL4-YFP2) under moderate light $\left(120 \mu \mathrm{mol} \mathrm{m} \mathrm{m}^{-2} \mathrm{~s}^{-1}\right)$. The comparative analysis revealed no significant differences in the amount of the tetrapyrrole intermediates and end product between WT, soul mutant, and a SOUL4 overexpressor line (Figure 4) (Venkatasalam, 2012).

\section{Casein Kinase II and Chloroplast Stroma Phosphorylate SOUL4 Protein In Vitro}

SOUL3 phosphorylation has been identified in the eyespot of $C$. reinhardtii (Wagner et al., 2008). We therefore examined SOUL4 phosphorylation in Arabidopsis. The C-terminus of SOUL4 has a conserved casein kinase II (CKII) target consensus sequence (S/ T-D/E-X-E/D) (Figure 5A) (Venkatasalam, 2012) and may therefore be a target of CKII like kinase. One of the five Arabidopsis CKII $\alpha$ subunits has been localized in the chloroplast stroma (Salinas et al., 2006). To determine whether recombinant SOUL4 is an in vitro substrate of CKII, we incubated recombinant SOUL4 with (Figure 5B, lane 2) or without (Figure 5B, lane 1) the recombinant maize CKII $\alpha$ subunit in a phosphorylation assay. To test whether SOUL4 is a potential substrate of the stromal CKII or other stromal kinases, the in vitro phosphorylation assay using recombinant SOUL4 was carried out with (Figure 5B, lane 3) or without (Figure 5B, lane 4) Arabidopsis stromal fraction (Venkatasalam, 2012). All the samples were incubated in the presence of $\left[\gamma^{33} \mathrm{P}\right]$ ATP. The samples were separated by SDS-PAGE followed by phosphorimager analysis, revealing phosphorylation of SOUL4 both by recombinant maize CKII and stromal fraction.

\section{DISCUSSION}

SOUL4 was synthesized as a $35 \mathrm{kDa}$ protein in vitro and converted to a $30 \mathrm{kDa}$, protease-resistant protein in an in vitro import assay into isolated chloroplasts. These results indicate that SOUL4 is synthesized as a cytosolic preprotein with a transit peptide $(\mathrm{N}$ terminal chloroplast targeting signal) that is cleaved upon import into chloroplasts. However, this does not provide any evidence regarding the suborganellar localization. Transient expression of a SOUL4-CFP fusion in tobacco leaves resulted in punctate fluorescence signals inside the chloroplast, indicative of a PG sublocalization. This was corroborated by colocalization with a well-accepted PG marker, FBN1a-YFP, and by co-distribution of SOUL4 with FBN2 by membrane fractionation on a sucrose gradient. Arabidopsis SOUL1, SOUL2, and SOUL5 homologs of SOUL4 are known examples of heme-binding proteins with well- 
$\mathbf{A}$

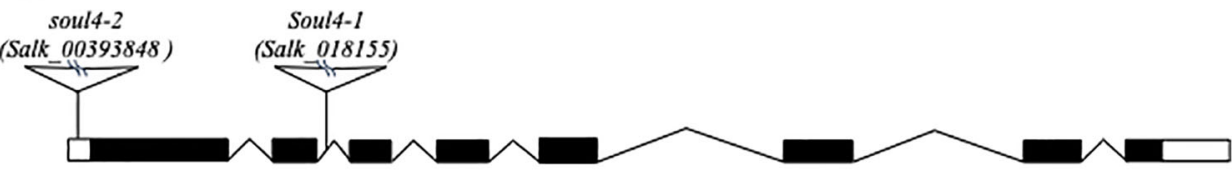

B

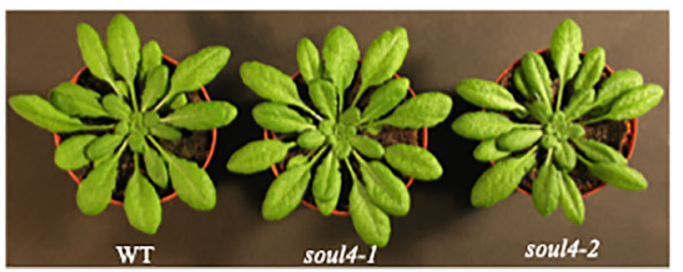

C

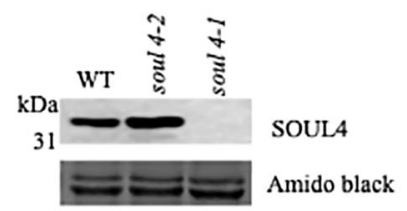

FIGURE 3 | Characterization of soul4 mutant in Arabidopsis. (A) Scheme showing the position of the T-DNA insertion in the SOUL4 gene. Introns are represented by black boxes and exons by black lines; 5'- and 3'-untranslated regions are shown as empty boxes. (B) Four-week-old wild type (Col-0), soul4-1, and soul4-2 plants were grown on soil. (C) Western blot analyses of total protein extracts for WT, soul4-2, and soul4-1 mutant were separated by SDS-PAGE, transferred to nitrocellulose membrane, and stained with Amido black for a loading control (lower panel). Antibody against SOUL4 was used for immunoblotting, as indicated (upper panel).

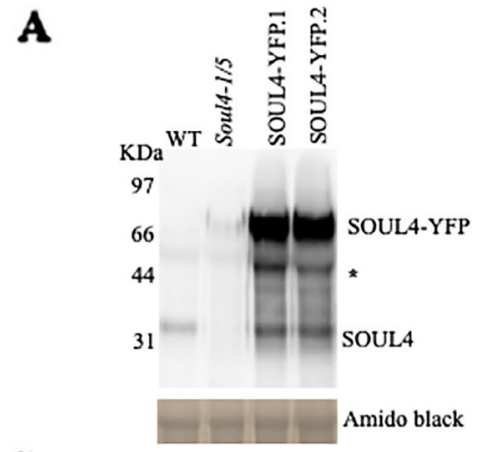

C

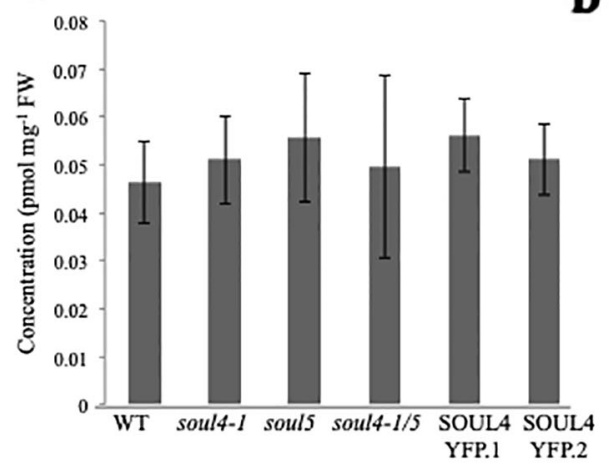

B
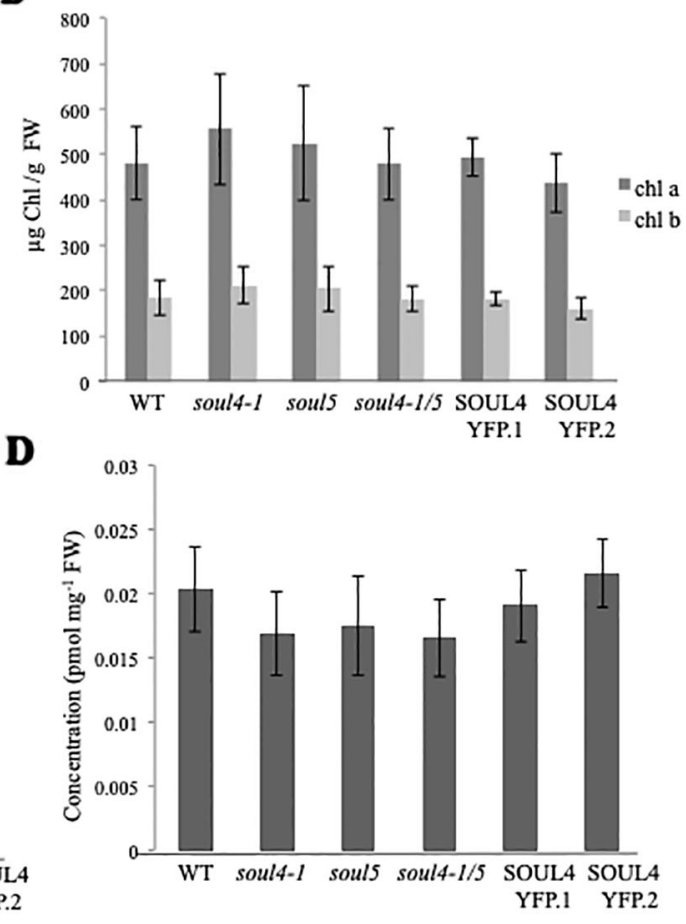

FIGURE 4 | Comparison of tetrapyrroles and chlorophyll pigment levels in soul mutants and SOUL4-YFP overexpressing lines. (A) Western blot analyses of total protein extracts for WT, soul4-1/5, and SOUL4-YFP overexpressing lines SOUL4-YFP.1 and SOUL4-YFP.2 were separated by SDS-PAGE, transferred to a nitrocellulose membrane, and stained with Amido black for a loading control (lower panel). Antibodies against SOUL4 were used for immunoblotting, as indicated (upper panel) and * indicated as a non-specific band. Chlorophyll a, b (B), Mg-protoporphyrin IX (C), and con-covalently bound heme (D) contents measured from wild type, soul4-1, soul5, soul4-1/5, and SOUL4-YFP overexpressing lines SOUL4-YFP.1 and SOUL4-YFP.2. The samples were extracted from 3-week-old Arabidopsis seedlings grown on soil under short-day condition. Data are averages of four biological replicates $( \pm \mathrm{SD})$. 
characterized biochemical properties (Takahashi et al., 2008; Lee et al., 2012). Purified recombinant SOUL4 (expressed without the transit peptide) bound to hemin-agarose in vitro, and the binding was competed by preincubation of SOUL 4 with free heme. This, in addition to the predicted tetrapyrrole-binding cleft, are strong indications that SOUL4 also acts as heme- or tetrapyrrolebinding protein in vivo. A number of known chloroplast metabolic enzymes and previously unclassified proteins are known to function in PG lipid metabolism [e.g. the VTE1 (Vidi et al., 2006), NDC1 (Eugeni Piller et al., 2011), and PES1 and 2 (Lippold et al., 2012)], and therefore, the potential role of SOUL4 in PG lipid metabolism was investigated using prenyllipid analysis. Under standard growth conditions, no significant differences in the lipid profiles of soul4-1 and WT were observed (Venkatasalam, 2012). However, this does not exclude that minor lipid components may have escaped detection or that major changes may be induced under stress conditions. SOUL4 has a conserved CKII target consensus sequence (S/T-D/E-X-E/D), and $C$. reinhardtii SOUL3 was present in the phosphoproteome of the eyespot that also contains other proteins conserved from PG (Wagner et al., 2008). A chloroplastic stromal CKII $\alpha$ subunit has been reported in mustard (Baginsky et al., 1999; Ogrzewalla et al., 2002) as well as Arabidopsis (Salinas et al., 2006). Chloroplastic CKII has a wide range of predicted substrates (Baginsky and Gruissem, 2009). Recombinant, purified SOUL4 was readily phosphorylated in vitro by maize CKII $\alpha$ subunit as well as an isolated stromal extract. The isolated extract is known to contain stromal CKII but most likely also other kinases. Therefore, it cannot be excluded that unknown stromal kinases other than CKII are able to phosphorylate SOUL4 in vitro. It appears likely that phosphorylation is a regulatory mechanism in SOUL4, but in view of the absence of a well-characterized function, its purpose

\section{$\mathbf{A}$}

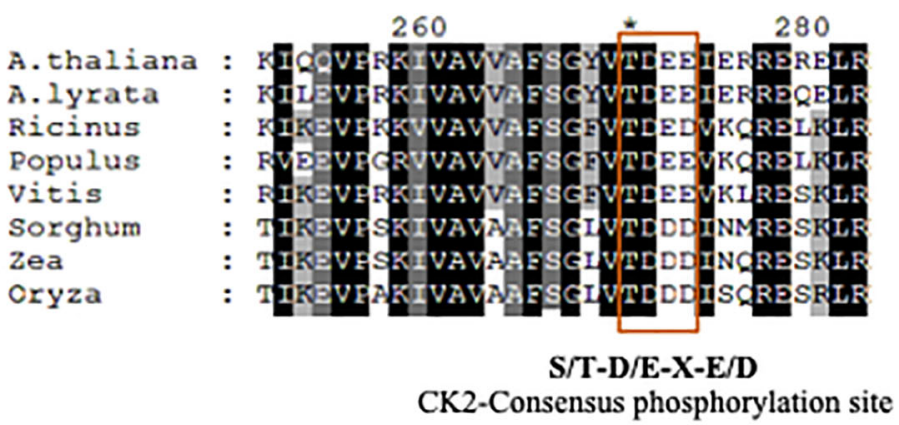

$\mathbf{B}$

FIGURE 5 | Phosphorylation of recombinant SOUL4 heme-binding protein by CK2 and stromal fractions (A) Alignment of SOUL4 with its homologs of other species indicating the predicted CKII phosphorylation site. SOUL4 from Arabidopsis thaliana NP_187624.1, A. Iyrata XP_002882654.1, Ricinus communis XP_002528755.1, Populus trichocarpa XP_002323500.1, Vitis vinifera XP_002282544.1, Sorghum bicolor XP_002452205.1, Zea mays NP_001147650.1, Oryza sativa NP_001047029.1 aligned by ClustalW. The C-terminal conserved casein kinase II (CKII) motif is highlighted and boxed. (B) Purified recombinant AtSOUL4 (3 $\mu$ g) was

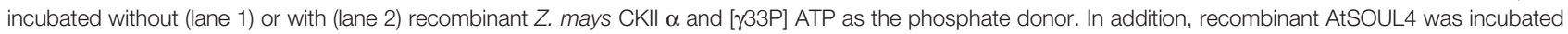
with (lane 3) or without (lane 4) isolated Arabidopsis stromal fraction. The samples were separated by SDS-PAGE followed by Coomassie blue staining (left panel) and phosphorimager analysis (right panel). The asterisk symbol (*) indicated as a specific band of phosphorylated AtSOUL4. 
remains unclear. The phenotypic analysis of the soul mutant and SOUL4 overexpressing lines grown on soil under standard conditions did not yield an apparent phenotype when compared to wild type. An exhaustive tetrapyrrole analysis suggested that under standard growth conditions, there was no significant difference in tetrapyrroles between WT, soul4-1, and SOUL4 overexpressing lines. Our results suggest that SOUL4 acts as heme-binding protein and may contribute to a pool of free heme molecules inside the chloroplast. The entire content of the covalently bound (mainly a- and c-type) and non-covalently bound (b-type) heme can currently not be determined. Methods are available to determine the non-covalently bound heme and more recently the free or labile heme portion (Weinstein and Beale, 1983; Masuda and Takahashi, 2006; Richter et al., 2019). The latter pool of heme is certainly only a subfraction of the non-covalently bound heme, but could likely include the heme portion bound to SOUL isoforms. It will be of future interest to determine hemebinding affinities of SOUL proteins and the dynamic of heme exchange of this group of proteins.

\section{MATERIALS AND METHODS}

\section{Plant Material and Growth}

Wild-type and mutant $A$. thaliana plants, ecotype Colombia, were used in all experiments. The soul4-1 (Salk_018155), soul4-2 (Salk_00393848), soul5 (SAIL_215_CO2), double mutant was generated by crossing soul4-1 and soul5 homozygous plants followed by genotyping (see Supplementary Table 1). Plants were grown either on soil under long-day conditions [16 h light $\left(120 \mu \mathrm{mol} \mathrm{m} \mathrm{m}^{-2}\right), 8 \mathrm{~h}$ dark, $21^{\circ} \mathrm{C}, 70 \%$ relative humidity] or on Murashige and Skoog (MS) medium under short-day conditions [ $8 \mathrm{~h}$ light $\left(120 \mu \mathrm{mol} \mathrm{m} \mathrm{m}^{-2} \mathrm{~s}^{-1}\right), 16 \mathrm{~h}$ dark, $21^{\circ} \mathrm{C}, 70 \%$ relative humidity).

\section{Localization Studies by \\ Fluorescent Marker}

The full length Arabidopsis SOUL4 cDNA was amplified using the SOUL4-F and SOUL4-R primers (see Supplementary Table 1) flanked by NcoI restriction sites and ligated into the NcoI site of the pCL62 vector, encoding a C-terminally CFP-tagged fusion protein. The pCL61 vector, encoding for the C-terminally YFP-tagged fusion protein of FBN1A, was used as PG marker. Both constructs were transformed into A. tumefaciens strain C58Ci. The SOUL4-CFP construct and the combination of SOUL4-CFP, FBN1A-YFP constructs were co-infiltrated into leaves of 2- to 3-week-old $N$. benthamiana plants. Infiltrated leaves were analyzed after 2 days under a Leica TCS SL confocal microscope. CFP and YFP were detected sequentially using 458 and $514 \mathrm{~nm}$ laser lines, as well as 460-510 and 520-588 nm detection windows, from a LEICA SP2 AOBS microscope. Chlorophyll autofluorescence was monitored using either $594 \mathrm{~nm}$ or TRITC (568 nm) excitation wavelengths. Images were captured and analyzed using LCS lite software (Leica).

\section{Chloroplast Isolation and In Vitro Import Reactions}

Arabidopsis plants were grown on MS medium as described. Intact chloroplasts were isolated according to Fitzpatrick and Keegstra, 2001.
Full length SOUL4 cDNA was amplified using the SOUL4-F and SOUL4-R primers (see Supplementary Table 1) flanked by NcoI restriction sites and ligated into the $\mathrm{NcoI}$ site of the pET21d vector. The pET21d-SOUL4 construct contains a T7 promoter upstream of the start codon used for in vitro protein synthesis using the TNT $^{\circledR}$ T7 Quick Coupled Transcription/Translation System (Promega Corporation, Madison WI, USA) as recommended by the supplier. Chloroplasts corresponding to $25 \mu \mathrm{g}$ chlorophyll were used for import assays. The import experiment of in vitro synthesized proteins into intact isolated chloroplasts has been described previously (Agne et al., 2009).

\section{Expression and Purification of Recombinant Proteins}

SOUL4 CDNA without the sequence encoding a transit peptide (72-309 amino acid) was amplified using the SOUL4-F(-TP) and SOUL4-R primers (see Supplementary Table 1) flanked by NcoI restriction sites and ligated into NcoI site of the pET21d vector, encoding for a $\mathrm{C}$-terminally $\mathrm{His}_{6}$-tagged fusion protein (pET21d-SOUL4-HIS). Mature SOUL4 protein was overexpressed in E. coli BL21 (DE3) transformed with the expression vector pET21d-SOUL4-HIS. Expression was induced using $0.4 \mathrm{mM}$ isopropyl- $\beta$-D-thiogalactopyranoside at $37^{\circ} \mathrm{C}$ for $3 \mathrm{~h}$. Bacterial pellets were lysed by sonication in 50 $\mathrm{mM}$ Tris- $\mathrm{HCl} \mathrm{pH} 8,150 \mathrm{mM} \mathrm{NaCl}$, and $5 \mathrm{mM}$ imidazol followed by centrifugation for $30 \mathrm{~min}$ at $14,000 \mathrm{rpm}$ (Sorvall, SS-34). The supernatant was filtered through a $0.45 \mu \mathrm{M}$ nitrocellulose filter, and the mature SOUL4-HIS protein was purified from the supernatant fraction by nickelnitrilotriacetic acid agarose affinity chromatography. Eluates were dialyzed against phosphate buffered saline (PBS). Glycerol (10\%) was added to the purified SOUL4-HIS protein and stored at $-80^{\circ} \mathrm{C}$.

\section{In Vitro Heme-Binding Assay}

The in vitro heme-binding assay was carried out as described (Vanhee et al., 2011). Recombinant SOUL4 protein $(100 \mu \mathrm{M})$ in PBS with glycerol was incubated with hemin-agarose (SigmaAldrich) or agarose beads for $1 \mathrm{~h}$ on a rotating wheel. The flowthrough was collected and the beads washed with PBS buffer and eluted with SDS-PAGE-loading buffer. For the heme competition assay, $100 \mu \mathrm{M}$ of recombinant mature SOUL4 protein in volume of $80 \mu \mathrm{l}$ was pre-incubated with $20 \mu \mathrm{l}$ of 10 $\mathrm{mM}$ hemin solution for $1 \mathrm{~h}$ before incubation with the heminagarose. The flow-through was collected and the beads washed with PBS buffer and eluted with SDS-PAGE-loading buffer. The input, flow-through, last wash, and elution were analyzed by SDS-PAGE and Western blot.

\section{Porphyrin and Heme Analysis}

Porphyrins were extracted and analyzed as described previously (Papenbrock et al., 2000). The non-covalently bound heme was extracted by acidic acetone containing $5 \% \mathrm{HCl}$, transferred to diethyl ether, concentrated, and washed on a DEAE-Sepharose column. Measurement of heme content has been described previously (Castelfranco and Jones, 1975). 


\section{Plant Transformation and Transgenic Lines}

The full length Arabidopsis SOUL4 cDNA was amplified using the SOUL4-AttB1 and SOUL4-AttB2 primers and ligated into the pENTR221 vector using BP clonase (Invitrogen) and inserted into the binary vector pEarleyGate101 using the LR clonase (Invitrogen), resulting in a vector coding for the SOUL4-YFP fusion under the control of the $35 \mathrm{~S}$ promoter. A. thaliana plants were transformed using the floral dip method as described (Clough and Bent, 1998).

\section{Protein Extraction and Immunoblotting}

Leaves were homogenized in $250 \mu \mathrm{l}$ of Rensink buffer [100 mM $\mathrm{NaCl}, 50 \mathrm{mM}$ Tris-HCl, pH 7.5, 1\% (v/v) Triton X-100, and $10 \mathrm{mM}$ $\beta$-mercaptoethanol] supplemented with protease inhibitor mixture (Sigma P9599) and centrifuged at 20,000 $\times$ g for $5 \mathrm{~min}$. Protein was quantified in the supernatant using the Bradford method. Proteins were concentrated by chloroform-methanol precipitation, separated by SDS-PAGE, and blotted onto nitrocellulose membrane. After protein transfer, the nitrocellulose membranes were stained with Amido black. Polyclonal anti-SOUL4 and antiFBN2 were obtained from rabbits immunized with recombinant SOUL4-HIS and FBN2-HIS protein (Eurogentec, Seraing, Belgium). Antibodies were further affinity-purified using recombinant protein coupled to Affi-gel10 (Bio-Rad Laboratories) according to the supplier's instructions. To probe the blots, primary antibodies recognizing SOUL4, FBN2, TOC75, and LHCB2 (Agrisera) were used.

\section{Phosphorylation Assays}

Three micrograms of recombinant SOUL4 protein were incubated with or without 37.5 units of recombinant maize (Zea mays) CK2 asubunit (Biaffin) and with or without stromal fractions in the presence of $25 \mathrm{mM}$ Tris- $\mathrm{HCl}, \mathrm{pH} 8.0,5 \mathrm{mM} \mathrm{MgCl} 2,1 \mathrm{mM}$ dithiothreitol (DTT), $50 \mathrm{mM}$ ATP, and $1 \mu \mathrm{Ci}$ of $[\gamma$-33P] ATP for $30 \mathrm{~min}$ at $25^{\circ} \mathrm{C}$. Reactions were stopped by diluting in ice-cold buffer followed by chloroform-methanol precipitation. The samples were separated by SDS-PAGE and examined by autoradiography.

\section{Chloroplast Membrane Fractionation and PG Isolation}

Chloroplasts membranes were isolated as previously reported (Vidi et al., 2006). Total membranes, corresponding to $10 \mathrm{mg}$ of

\section{REFERENCES}

Agne, B., Infanger, S., Wang, F., Hofstetter, V., Rahim, G., Martin, M., et al. (2009). A toc159 import receptor mutant, defective in hydrolysis of GTP, supports preprotein import into chloroplasts. J. Biol. Chem. 284, 8670-8679. doi: 10.1074/jbc.M804235200

Austin, J. R.2nd, Frost, E., Vidi, P. A., Kessler, F., and Staehelin, L. A. (2006). Plastoglobules are lipoprotein subcompartments of the chloroplast that are permanently coupled to thylakoid membranes and contain biosynthetic enzymes. Plant Cell 18, 1693-1703. doi: 10.1105/tpc.105.039859

Baginsky, S., and Gruissem, W. (2009). The chloroplast kinase network: new insights from large-scale phosphoproteome profiling. Mol. Plant 2, 1141-1153. doi: $10.1093 / \mathrm{mp} / \mathrm{ssp} 058$ chlorophyll, were sedimented at 100,000 $\times$ g and resuspended in $2 \mathrm{ml} 45 \%$ sucrose in TE buffer. Membranes were overlaid with a linear sucrose gradient (5-45\% sucrose) and centrifuged for $17 \mathrm{~h}$ at $100,000 \times \mathrm{g}$ and $4^{\circ} \mathrm{C}$ (SW41Ti rotor, Beckman). Fractions $(1 \mathrm{ml})$ were collected starting from the top of the gradient and used for Western blotting.

\section{DATA AVAILABILITY STATEMENT}

The datasets generated for this study are available on request to the corresponding author.

\section{AUTHOR CONTRIBUTIONS}

VS and FK designed the experiments. BG contributed the tetrapyrrole and chlorophyll analyses. VS performed all experiments. VS, FK, and BG analyzed the data, and VS wrote the manuscript with the help of FK and BG.

\section{FUNDING}

This work was supported by the Swiss National Science Foundation (SNSF) grants 31003A_156998 and 31003A_176191. Work in BG's laboratory was partially supported by the Deutsche Forschungsgemeinschaft (Subproject C04 of the DFG SFB/TR175).

\section{ACKNOWLEDGMENTS}

VS thanks the University of Neuchâtel for archiving his $\mathrm{PhD}$ thesis online. Part of his thesis was used and referenced in this manuscript.

\section{SUPPLEMENTARY MATERIAL}

The Supplementary Material for this article can be found online at: https://www.frontiersin.org/articles/10.3389/fpls.2020.00002/ full\#supplementary-material

Baginsky, S., Tiller, K., Pfannschmidt, T., and Link, G. (1999). PTK, the chloroplast RNA polymerase-associated protein kinase from mustard (Sinapis alba), mediates redox control of plastid in vitro transcription. Plant Mol. Biol. 39, 1013-1023. doi: 10.1023/A:1006177807844

Balestrasse, K. B., Noriega, G. O., Batlle, A., and Tomaro, M. L. (2005). Involvement of heme oxygenase as antioxidant defense in soybean nodules. Free Radic. Res. 39, 145-151. doi: 10.1080/10715760400022319

Brzezowski, P., Richter, A. S., and Grimm, B. (2015). Regulation and function of tetrapyrrole biosynthesis in plants and algae. Biochim. Biophys. Acta 1847, 968-985. doi: 10.1016/j.bbabio.2015.05.007

Busch, A. W., and Montgomery, B. L. (2015). Interdependence of tetrapyrrole metabolism, the generation of oxidative stress and the mitigative oxidative stress response. Redox Biol. 4, 260-271. doi: 10.1016/j.redox.2015.01.010 
Castelfranco, P. A., and Jones, O. T. (1975). Protoheme turnover and chlorophyll synthesis in greening barley tissue. Plant Physiol. 55, 485-490. doi: 10.1104/ pp.55.3.485

Clough, S. J., and Bent, A. F. (1998). Floral dip: a simplified method for Agrobacterium-mediated transformation of Arabidopsis thaliana. Plant J. 16, 735-743. doi: 10.1046/j.1365-313x.1998.00343.x

Deruére, J., Römer, S., d'Harlingue, A., Backhaus, R. A., Kuntz, M., and Camara, B. (1994). Fibril assembly and carotenoid overaccumulation in chromoplasts: a model for supramolecular lipoprotein structures. Plant Cell 6, 119-133. doi: 10.1105/tpc.6.1.119

Dias, J. S., Macedo, A. L., Ferreira, G. C., Peterson, F. C., Volkman, B. F., and Goodfellow, B. J. (2006). The first structure from the SOUL/HBP family of heme-binding proteins, murine P22HBP. J. Biol. Chem. 281, 31553-31561. doi: 10.1074/jbc.M605988200

Espinas, N. A., Kobayashi, K., Takahashi, S., Mochizuki, N., and Masuda, T. (2012). Evaluation of unbound free heme in plant cells by differential acetone extraction. Plant Cell Physiol. 53, 1344-1354. doi: 10.1093/pcp/pcs067

Eugeni Piller, L., Besagni, C., Ksas, B., Rumeau, D., Brehelin, C., Glauser, G., et al. (2011). Chloroplast lipid droplet type II NAD(P)H quinone oxidoreductase is essential for prenylquinone metabolism and vitamin K1 accumulation. Proc. Natl. Acad. Sci. U. S. A 108, 14354-14359. doi: 10.1073/pnas.1104790108

Fitzpatrick, L. M., and Keegstra, K. (2001). A method for isolating a high yield of Arabidopsis chloroplasts capable of efficient import of precursor proteins. Plant J. 27, 59-65. doi: 10.1046/j.0960-7412.2001.01061.x

Kessler, F., Schnell, D., and Blobel, G. (1999). Identification of proteins associated with plastoglobules isolated from pea (Pisum sativum L.) chloroplasts. Planta 208, 107-113. doi: 10.1007/s004250050540

Kobayashi, K., and Masuda, T. (2016). Transcriptional regulation of tetrapyrrole biosynthesis in Arabidopsis thaliana. Front. Plant Sci. 7, 1811. doi: 10.3389/ fpls.2016.01811

Lee, H. J., Mochizuki, N., Masuda, T., and Buckhout, T. J. (2012). Disrupting the bimolecular binding of the haem-binding protein 5 (AtHBP5) to haem oxygenase 1 (HY1) leads to oxidative stress in Arabidopsis. J. Exp. Bot. 63, 5967-5978. doi: 10.1093/jxb/ers242

Lippold, F., Vom Dorp, K., Abraham, M., Holzl, G., Wewer, V., Yilmaz, J. L., et al. (2012). Fatty acid phytyl ester synthesis in chloroplasts of Arabidopsis. Plant Cell 24, 2001-2014. doi: 10.1105/tpc.112.095588

Lundquist, P. K., Poliakov, A., Bhuiyan, N. H., Zybailov, B., Sun, Q., and Van Wijk, K. J. (2012). The functional network of the Arabidopsis plastoglobule proteome based on quantitative proteomics and genome-wide coexpression analysis. Plant Physiol. 158, 1172-1192. doi: 10.1104/pp.111.193144

Masuda, T., and Takahashi, S. (2006). Chemiluminescent-based method for heme determination by reconstitution with horseradish peroxidase apo-enzyme. Anal. Biochem. 355, 307-309. doi: 10.1016/j.ab.2006.04.008

Merchant, S. S., Prochnik, S. E., Vallon, O., Harris, E. H., Karpowicz, S. J., Witman, G. B., et al. (2007). The Chlamydomonas genome reveals the evolution of key animal and plant functions. Science 318, 245-250. doi: 10.1126/ science. 1143609

Mochizuki, N., Tanaka, R., Grimm, B., Masuda, T., Moulin, M., Smith, A. G., et al. (2010). The cell biology of tetrapyrroles: a life and death struggle. Trends Plant Sci. 15, 488-498. doi: 10.1016/j.tplants.2010.05.012

Ogrzewalla, K., Piotrowski, M., Reinbothe, S., and Link, G. (2002). The plastid transcription kinase from mustard (Sinapis alba L.). A nuclear-encoded CK2type chloroplast enzyme with redox-sensitive function. Eur. J. Biochem. 269, 3329-3337. doi: 10.1046/j.1432-1033.2002.03017_269_13.x

Papenbrock, J., Mock, H. P., Tanaka, R., Kruse, E., and Grimm, B. (2000). Role of magnesium chelatase activity in the early steps of the tetrapyrrole biosynthetic pathway. Plant Physiol. 122, 1161-1169. doi: 10.1104/pp.122.4.1161

Peltier, J. B., Cai, Y., Sun, Q., Zabrouskov, V., Giacomelli, L., Rudella, A., et al. (2006). The oligomeric stromal proteome of Arabidopsis thaliana chloroplasts. Mol. Cell Proteomics 5, 114-133. doi: 10.1074/mcp.M500180-MCP200

Pozueta-Romero, J., Rafia, F., Houlnè, G., Cheniclet, C., Carde, J. P., Schantz, M. L., et al. (1997). A ubiquitous plant housekeeping gene, PAP, encodes a major protein component of bell pepper chromoplasts. Plant Physiol. 115, 11851194. doi: 10.1104/pp.115.3.1185
Richter, A. S., Banse, C., and Grimm, B. (2019). The GluTR-binding protein is the heme-binding factor for feedback control of glutamyl-tRNA reductase. Elife 8, 1-18. doi: 10.7554/eLife.46300

Salinas, P., Fuentes, D., Vidal, E., Jordana, X., Echeverria, M., and Holuigue, L. (2006). An extensive survey of CK2 a and Bsubunits in Arabidopsis: multiple isoforms exhibit differential subcellular localization. Plant Cell Physiol. 47, 1295-1308. doi: 10.1093/pcp/pcj100

Sato, E., Sagami, I., Uchida, T., Sato, A., Kitagawa, T., Igarashi, J., et al. (2004). SOUL in mouse eyes is a new hexameric heme-binding protein with characteristic optical absorption, resonance Raman spectral, and hemebinding properties. Biochemistry 43, 14189-14198. doi: 10.1021/bi048742i

Schulze, T., Schreiber, S., Iliev, D., Boesger, J., Trippens, J., Kreimer, G., et al. (2013). The heme-binding protein SOUL3 of Chlamydomonas reinhardtii influences size and position of the eyespot. Mol. Plant 6, 931-944. doi: 10.1093/ $\mathrm{mp} / \mathrm{sss} 137$

Takahashi, S., Ogawa, T., Inoue, K., and Masuda, T. (2008). Characterization of cytosolic tetrapyrrole-binding proteins in Arabidopsis thaliana. Photochem. Photobiol. Sci. 7, 1216-1224. doi: 10.1039/b802588f

Van Wijk, K. J., and Kessler, F. (2017). Plastoglobuli: plastid microcompartments with integrated functions in metabolism, plastid developmental transitions, and environmental adaptation. Annu. Rev. Plant Biol. 68, 253-289. doi: 10.1146/annurev-arplant-043015-111737

Vanhee, C., Zapotoczny, G., Masquelier, D., Ghislain, M., and Batoko, H. (2011). The Arabidopsis multistress regulator TSPO is a heme binding membrane protein and a potential scavenger of porphyrins via an autophagy-dependent degradation mechanism. Plant Cell 23, 785-805. doi: 10.1105/tpc.110.081570

Veenman, L., Vainshtein, A., Yasin, N., Azrad, M., and Gavish, M. (2016). Tetrapyrroles as endogenous TSPO ligands in eukaryotes and prokaryotes: Comparisons with synthetic ligands. Int. J. Mol. Sci. 17, 1-26. doi: 10.3390/ ijms 17060880

Venkatasalam, S. (2012). Plastoglobules - A new destination for recombinant proteins produced in transplastomic plants and characterization of plastidial At-SOUL heme binding protein. [dissertation] ([Neuchâtel]: University of Neuchâtel).

Vidi, P. A., Kanwischer, M., Baginsky, S., Austin, J. R., Csucs, G., Dormann, P., et al. (2006). Tocopherol cyclase (VTE1) localization and vitamin E accumulation in chloroplast plastoglobule lipoprotein particles. J. Biol. Chem. 281, 11225-11234. doi: 10.1074/jbc.M511939200

Wagner, V., Ullmann, K., Mollwo, A., Kaminski, M., Mittag, M., and Kreimer, G. (2008). The phosphoproteome of a chlamydomonas reinhardtii eyespot fraction includes key proteins of the light signaling pathway. Plant Physiol. 146, 772-788. doi: 10.1104/pp.107.109645

Weinstein, J. D., and Beale, S. I. (1983). Separate physiological roles and subcellular compartments for two tetrapyrrole biosynthetic pathways in Euglena gracilis. J. Biol. Chem. 258, 6799-6807.

Ytterberg, A. J., Peltier, J. B., and Van Wijk, K. J. (2006). Protein profiling of plastoglobules in chloroplasts and chromoplasts. A surprising site for differential accumulation of metabolic enzymes. Plant Physiol. 140, 984-997. doi: 10.1104/pp.105.076083

Zylka, M. J., and Reppert, S. M. (1999). Discovery of a putative heme-binding protein family (SOUL/HBP) by two-tissue suppression subtractive hybridization and database searches. Brain Res. Mol. Brain Res. 74, 175-181. doi: 10.1016/S0169-328X(99)00277-6

Conflict of Interest: The authors declare that the research was conducted in the absence of any commercial or financial relationships that could be construed as a potential conflict of interest.

Copyright (c) 2020 Shanmugabalaji, Grimm and Kessler. This is an open-access article distributed under the terms of the Creative Commons Attribution License (CC BY). The use, distribution or reproduction in other forums is permitted, provided the original author(s) and the copyright owner(s) are credited and that the original publication in this journal is cited, in accordance with accepted academic practice. No use, distribution or reproduction is permitted which does not comply with these terms. 